\title{
A IMPORTÂNCIA DO CUIDADOR NO CONTEXTO DA SAÚDE DO IDOSO
}

\author{
The Importance of the Caregiver \\ in the Elderly Health Context \\ La Importancia del Cuidador en el \\ Contexto de la Salud del Anciano
}

Marcia Duarte Moreira

Célia Pereira Caldas

\section{Resumo}

Trata-se de um estudo bibliográfico que busca discutir a temática "idoso dependente de cuidados e a capacitação do cuidador no contexto domiciliar". A análise foi realizada a partir de autores que desenvolveram estudos sobre envelhecimento com dependência, cuidado familiar e ações direcionadas ao cuidador informal. Os temas destacados para esta análise foram: o papel do cuidador informal no cuidado do idoso dependente; a vivência de ser um cuidador familiar; e os modelos de atenção e suporte direcionados ao cuidador informal. 0 estudo demonstra a importância da ampliação de estratégias que tenham o cuidador como sujeito principal, cabendo ao profissional de saúde e às políticas públicas valorizarem a rede de suporte ao idoso dependente. Esta rede é importante como base do processo de cuidar com qualidade.

Palavras-chave: Cuidadores. Idoso. Família.

Abstract

The present study is a bibliographic research that aims to discuss the theme "the dependent elderly and caregiver's skills in domiciliary context". The authors who developed studies on dependent elderly, familiar care and nonprofessional care actions were consulted. The themes emphasized were: the role of non-professional caregivers; the experience of caring a relative, and models of assistance and support for non-professional caregivers. The study shows the importance of establishing strategies of support for the community Care. It is up to the health professionals and to the public policies to value a support system to the dependent aged cared in community. This system should be the base for a qualified process of care.

\section{Resumen}

Se trata de un estudio bibliográfico que busca discutir la tematica del anciano dependiente y la capacitación del cuidador en el contexto domiciliar. La análisis fue realizada a partir de autores que desarollaron estudios sobre envejecimiento con dependencia, cuidado familiar y acciones direccionadas al cuidador informal. Los temas destacados para esta análisis fueron: el papel del cuidador informal en el cuidado al idoso dependiente; la vivencia de ser un cuidador familiar; y los modelos de atención y suporte dirigidos para el cuidador informal. El estudio muestra la importancia de la ampliación de estrategias que tengan el cuidador como sujeto principal, siendo de competencia del profesional de salud y de las políticas públicas valoraren la red de soporte al idoso dependiente y tenerla como aliada en el proceso de cuidar con calidad. 


\section{INTRODUÇÃO}

Trata-se de um estudo na área de enfermagem gerontogeriátrica que tem como temática o idoso dependente de cuidados e a capacitação do cuidador no contexto domiciliar.

0 envelhecimento populacional brasileiro vem se acentuando consideravelmente, gerando impactos nas diversas formas de se prestar cuidados ao grupo idoso. Segundo dados da Organização Mundial de Saúde (OMS), entre 1950 e 2025, a população de idosos no país crescerá 16 vezes contra 5 vezes o crescimento populacional total, colocando nosso país como a sexta população em contingente de idosos no mundo'.

Um fenômeno a se destacar é o crescimento da população "mais idosa", considerada acima de 80 anos, trazendo como conseqüência alterações na própria composição etária dentro do grupo de idosos. Este fato nos permite observar pessoas na faixa de 60 anos "em pleno vigor físico e mental, bem como pessoas na faixa de 90 anos, que devem se encontrar em maior vulnerabilidade"2.

Aproximadamente $40 \%$ dos idosos que apresentam idade entre 75 e 84 anos e mais da metade da população de 85 anos e mais apresentam algum grau de incapacidade 3 . Isso gera cada vez mais um quadro de sobrevivência de idosos na dependência de pessoas para suprirem suas incapacidades, e, na maioria das vezes, essas pessoas são os familiares ${ }^{4}$.

Definimos incapacidade como a deficiência de um órgão ou sistema sobre o funcionamento do indivíduo em termos de limitações de função ou de restrição de atividades ${ }^{5}$. A dependência consiste na condição que faz com que a pessoa necessite do auxílio de outrem para a realização de atividades do seu dia-a-dia ${ }^{6}$.

Ao contrário dos países desenvolvidos que fornecem uma rede estatal de apoio ao idoso dependente, em países menos desenvolvidos, como o Brasil, que ainda apresenta carências na Saúde Pública e Seguridade Social ${ }^{7}$, a família constitui o principal núcleo de apoio social.

Com o processo de envelhecimento da população e conseqüente aumento do número de idosos que vivenciam um processo de doença crônica e incapacitante, tornando-se dependentes, vem crescendo a preocupação sobre 0 cuidador familiar que, por vezes, não se encontra adequadamente preparado para essa prática, podendo acarretar processos deletérios no cuidar desses idosos.

0 familiar vivencia a sobrecarga física, emocional e socioeconômica, e é fundamental o treinamento adequado para que ele se torne mais seguro e preparado para assumir as responsabilidades no cuidado do familiar dependente ${ }^{8}$.

Partindo dessa premissa, foi estabelecida como estratégia norteadora deste trabalho bibliográfico a busca de estudos que enfocam as propostas e ações direcionadas à capacitação do cuidador do idoso dependente no contexto domiciliar. Assim, os objetivos são discutir a importância do papel do cuidador informal no cuidado do idoso dependente; compreender o papel vivenciado por esse familiar, focalizando-o como sujeito ativo do processo do cuidar; e avaliar as ações e propostas direcionadas a esse cuidador.

A relevância desse tema se destaca porque ainda não existem políticas oficiais direcionadas para o cuidado à população idosa que desenvolve dependência funcional e não há o reconhecimento dos cuidadores de idosos na atenção cotidiana?.

Este estudo, portanto, vai ao encontro das diretrizes estabelecidas pela Política Nacional de Saúde do Idoso (1999), que estimula discussões e estudos sobre o envelhecimento com dependência, inserindo nesse contexto o cuidador informal, sujeito indispensável para uma parceria adequada no cuidado ao idoso, com vistas a estimular a qualidade dessa assistência, buscando a reflexão sobre nossa prática junto a esse indivíduo.

\section{MÉTODO}

Este estudo caracterizou-se como sendo de natureza descritiva, retrospectiva, de revisão bibliográfica acerca da importância do cuidador no contexto saúde do idoso. Utilizamos como descritores os termos cuidadores, idosos, cuidados familiares, efetuando a consulta na base de dados MEDLINE, BIREME e LILACS. Definimos como critérios de inclusão as publicações dos últimos cinco anos sobre a temática proposta.

\section{RESULTADOS}

A partir do levantamento realizado, foram identificados, no intervalo compreendido entre os anos de 2000 e 2005, 31 trabalhos, sendo 22 artigos publicados nos periódicos selecionados e 9 capítulos de livros.

0 critério de categorização utilizado obedeceu aos objetivos propostos, sendo compostas três categorias que resultaram no desenvolvimento do artigo: o cuidador informal no contexto saúde do idoso; a vivência de ser um cuidador familiar e os modelos de atenção e suporte direcionados ao cuidador informal.

\section{O PAPEL DO CUIDADOR INFORMAL DO IDOSO DEPENDENTE}

No Brasil, a Constituição de 1988 considera responsabilidade da família, da sociedade e do Estado 0 suporte ao idoso, além do fornecimento de subsídios que garantam sua participação na comunidade, a defesa de sua dignidade e bem-estar e a garantia do direito à vida.

Novas leis e medidas foram empreendidas pelo Estado com o objetivo de proteger a pessoa idosa contra a discriminação, a violência e as dificuldades. A Política Nacional do Idoso (1994) e o Estatuto do Idoso são exemplos dessas medidas legais ${ }^{10}$.

No plano de atenção à saúde, somente em 1999, 0 Ministério da Saúde elaborou a Política Nacional de Saúde do Idoso, pois os altos custos envolvidos no tratamento dos idosos pelo Sistema Único de Saúde (SUS) não estavam resultando em ações efetivas na garantia da resolução das reais necessidades desse público ${ }^{11}$.

Em países como o Brasil, onde existem deficiências no setor público, particularmente nas áreas de Saúde Pública e Seguridade Social, a família continua representando fonte primordial de assistência para parcela significativa da população idosa.

Considerando o quadro de redução dos recursos estatais, da desmontagem do sistema de proteção social e de dificuldades de emprego, o envelhecimento da população do país tem sido 
visto como uma sobrecarga para as famílias, sendo essa prática reforçada pela queda da fecundidade e pela maior participação das mulheres no mercado de trabalho ${ }^{3}$.

Muitos idosos dependentes não podem nem devem ser mantidos em instituições hospitalares, principalmente quando não necessitam mais dos cuidados médico-hospitalares específicos, apesar de ainda necessitarem de cuidados especiais devido à dependência ${ }^{12}$.

Na maioria dos casos, pela falta de recursos de ordem financeira que permitam a contratação de cuidadores especializados no ambiente domiciliar, esses cuidados são realizados por um membro da família, com exceção dos idosos sem rede de apoio familiar, que também constituem um grande problema a ser destacado.

Cuidador é a pessoa, membro ou não da família, que, com ou sem remuneração, cuida do idoso doente ou dependente no exercício de suas atividades diárias, tais como alimentação, higiene pessoal, medicação de rotina, acompanhamento aos serviços de saúde e demais serviços requeridos do cotidiano - como a ida a bancos ou farmácias - , excluídas as técnicas ou procedimentos identificados com profissões legalmente estabelecidas, particularmente na área da enfermagem ${ }^{6}$.

A família é, geralmente, a primeira e a mais constante unidade de saúde para seus membros, sendo que o cuidado prestado envolve ações de promoção da saúde, prevenção e tratamento de doenças, incluindo as de reabilitação ${ }^{13}$.

Geralmente a função de cuidador é assumida por uma única pessoa, denominada cuidador principal, seja por instinto, vontade, disponibilidade ou capacidade ${ }^{14}$.

Em um estudo ${ }^{15}$ com pacientes e cuidadores assistidos pelo serviço Pós-Alta-Hospitalar de um hospital no Rio Grande do Sul, foram destacados três tipos de cuidadores: cuidadores dedicados - estão sempre disponíveis e preocupados com quem cuidam, fazendo questão de mostrar e avaliar seu cuidado; cuidadores por obrigação - cuidam do familiar por não haver outro que os substitua. Não se mostram disponíveis e queixamse da tarefa de cuidar; cuidadores sem iniciativa - quase não se envolvem e participam das orientações que possam contribuir para melhorar o bem-estar do idoso.

Em relação ao perfil do cuidador, autores ${ }^{16} \mathrm{em} 2002$ constataram o predomínio de mulheres cuidadoras (73\%), especialmente esposas e filhas, predominando também os arranjos com duas ou mais gerações, e que apenas $6 \%$ dos idosos viviam sozinhos.

Em 2004, um estudo ${ }^{17}$ com cuidadores de idosos de um serviço psicogeriátrico obteve como resultados que a maioria dos cuidadores era do sexo feminino, com média de idade de 51,3 anos, sem emprego ou do lar.

Um ponto a ser destacado é que a faixa etária dos cuidadores pertence, na maioria das vezes, à mesma geração das pessoas que cuidam, ou seja, "são idosos jovens independentes cuidando de idosos dependentes".

Os familiares se constituem numa rede autônoma de atendimento ao idoso, sem a devida integração com os serviços de saúde ${ }^{14}$ e muitas vezes desconhecem que poderiam recorrer aos profissionais e aos serviços na busca de apoio e orientações ${ }^{18}$.
Consiste em um desafio para os profissionais de saúde conhecer mais as famílias, já que, nas situações de doença de um membro familiar, as primeiras medidas são tomadas ainda no ambiente doméstico, sendo este o local onde se processa o principal cuidado ${ }^{15}$.

\section{A VIVÊNCIA DE SER UM CUIDADOR FAMILIAR}

A família funciona, na maioria dos casos, como uma totalidade, onde cada indivíduo desempenha um papel que irá influenciar no todo. A partir do momento em que um membro desse grupo adoece e não cumpre mais esse papel definido, a organização anterior sofre uma alteração que desencadeia uma crise, obrigando à reestruturação de papéis ${ }^{18}$.

Relações conjugais e relacionamentos entre pais e filhos envolvem uma complexa rede de sentimentos que funciona de modo circular na tentativa de manter um equilibrio. Quando um desses sujeitos adoece, cuidar pode ser uma forma de manter um vínculo $0^{18}$.

Em estudo realizado com um grupo de cuidadores no Rio Grande do Sul, os entrevistados referiram ter assumido o cuidado por obrigação matrimonial, e isso ocorria até com os idosos que não eram casados, mas que viviam juntos ${ }^{14}$.

Se o cônjuge não pode realizar o cuidado, o papel é assumido pelos filhos, mas também por outras figuras como os netos ${ }^{14}$.

A família, diante do idoso necessitado de cuidado ao longo da vida, pela cronicidade de sua situação de saúde, vivencia sentimentos de culpa, frustração, amor e solidariedade, experimentando um processo de construção da aceitação do fato que atravessa os caminhos da negação, entrega à dor, podendo gerar depressão e suas consequêências deletérias ${ }^{18}$.

A perda da identidade do cuidador pode acontecer, pois o idoso altera o seu papel na família, ocasionando novas ações a serem assumidas pelo familiar. "A mudança drástica nos relacionamentos ameaça o selfaté que se descubram outras maneiras de viver"18.

Vale destacar também que a carência, ou até mesmo a ausência do cuidado por uma dessas partes, pode ser fruto de relacionamentos e vínculos desfeitos por situações desagradáveis ou até mesmo vínculos que nunca chegaram a se estabelecer.

Através da experiência com um grupo de cuidadores familiares de idosos dependentes no ambulatório do Núcleo de Atenção ao Idoso (NAl), foi descrito em $2000^{18}$ o perfil dos cuidadores como sendo pessoas engajadas na solução dos problemas, sendo mais comuns os casos de monopolização da função do cuidador.

0 familiar que direciona para si todas as atividades relacionadas ao cuidado pode manifestar sentimentos de desconforto e solidão pela falta de apoio dos familiares, gerando uma crise no seu desempenho, "em que a habitual relação de afeto e de reciprocidade... é substituída por uma relação em que predomina, de forma unilateral, a imperiosa necessidade de fazer pelo outro... praticamente sem nenhum retorno pessoal" ${ }^{\prime \prime}$.

Estudos de $2003^{20}$ com cuidadores de idosos que sofreram acidente vascular cerebral (AVC) destacaram aspectos como 0 isolamento social decorrente do acúmulo de tarefas, perda do companheiro nas atividades sociais (para os cônjuges cuidadores), distúrbios comportamentais do portador de AVC, alterações no relacionamento da família e de amizades, sendo 
ressaltada ainda a sobrecarga psicológica, nem sempre exteriorizada, mas que se apresenta com sentimentos de ansiedade, insegurança e medo.

Em contrapartida, autores em $2004^{12}$ afirmam que 0 cuidador, apesar de vivenciar a sobrecarga, pode experimentar sentimentos de prazer e conforto quando se envolve no cuidar e vê que sua prática tem resultados positivos no tocante à melhora do idoso. Além disso, ao cuidar de um idoso dependente, o cuidador depara-se com o seu próprio processo de envelhecimento e com sua própria finitude.

Problemas no âmbito financeiro merecem destaque, pois estima-se que mais de $14 \%$ dos cuidadores desistem de seus empregos para se dedicarem exclusivamente aos seus idosos ${ }^{20} \mathrm{e}$, por tentarem suprir num primeiro momento as necessidades do idoso, acabam deixando os outros gastos para um segundo plano ${ }^{13}$.

Em um estudo realizado em São Paulo ${ }^{21}$, constatou-se que mais de $90 \%$ das famílias não recebem auxílio de serviços, organizações ou grupos voluntários/agências particulares, mas que cerca de $30 \%$ gostariam de receber ajuda financeira.

Pouco se conhece sobre o impacto econômico da dependência do idoso na família e no sistema de saúde associado à falência de nosso sistema previdenciário que transfere todo o encargo econômico do cuidar para a família ${ }^{21}$.

Segundo pesquisa com esposas cuidadoras de idosos dependentes participantes do projeto Bambuí em Minas Gerais (estudo populacional associado à necessidade de cuidador entre idosos), foi apontado que as tarefas mais cansativas, e que iam se tornando mais pesadas com a evolução da doença, se relacionavam às mais íntimas, como o banho, a higiene íntima e os cuidados com as feridas ${ }^{5}$.

A dificuldade em lidar com a doença pela falta de compreensão da sua magnitude foi um tema muito explorado em um estudo de $2004^{19}$ realizado com cuidadores informais de idosos portadores de doença de Alzheimer (DA) em Porto Alegre.

Os cuidadores apontaram dificuldades em obter informações para entender e enfrentar a doença de seu familiar. A consulta médica, na maioria das vezes, é centrada na patologia, deixando o cuidador sem a necessária orientação, sendo em casa que o familiar irá aprender "à medida que os problemas vão aparecendo" ${ }^{19}$.

Corroborando essa afirmativa, outras pesquisas ${ }^{17}$ enfatizam que a grande maioria da população de cuidadores informais no país ainda se encontra sem as informações e o suporte necessários de assistência ao idoso dependente.

0 sistema de saúde pública nacional não está preparado para dar suporte ao idoso que adoece nem à família que cuida, gerando o aumento da sobrecarga e o estresse ${ }^{19,22}$.

Fica a impressão de que estão ambos esquecidos, cuidador e quem precisa de cuidados, vivendo entre quatro paredes e, ao seu modo, vivenciando a dura realidade da incapacidade funcional numa sociedade que não parece acreditar que está envelhecendo, não cria aparelhos para fazer face ao desafio que representa cuidar de idosos que não envelheceram com saúde ${ }^{5}$.

\section{MODELOS DE ATENÇÃO E SUPORTE DIRECIONADOS AO CUIDADOR INFORMAL}

A literatura vem demonstrando que a tarefa de cuidar de idosos dependentes pode ocasionar efeitos adversos, gerando impactos negativos e sobrecarga no âmbito físico, psicológico, social e financeiro, devendo ser desenvolvidos programas destinados a prevenir esses efeitos e a trabalhar na busca da qualidade de vida do cuidador $^{16}$.

As necessidades das famílias relacionadas ao cuidado ao idoso dependente são, de um modo geral, de orientação sobre como deve ser desenvolvido esse cuidado, como ocorre o tratamento do processo que gera a dependência, além da necessidade de poder compartilhar as dúvidas e outras questões com os profissionais ${ }^{22}$.

0 sucesso do cuidado ao idoso no domicílio depende de quem irá realizá-lo e o quão preparado estará esse familiar. Cabe ao profissional de saúde avaliar a rede de suporte do idoso antes de conceder-lhe a alta hospitalar, sendo necessário o acompanhamento domiciliar para a avaliação do cuidado e do suporte adequado ${ }^{23}$.

Os cuidados de saúde no país possuem caráter individual e curativo, fragmentado em especialidade e profissionais, gerando a necessidade de uma reorganização dos serviços de saúde para que esses profissionais adotem estratégias de apoio às famílias ${ }^{19}$.

No Brasil, apesar da Política Nacional do Idoso (PNI), não há um programa de governo direcionado para o idoso dependente e embora seja apontado pela Constituição Federal, pela Política Nacional do Idoso e pela Política Nacional de Saúde do Idoso que a família é responsável pelo cuidado do idoso em relação ao atendimento de suas necessidades, não há um sistema de apoio às famílias e definição das responsabilidades das instâncias de cuidados formais e informais na prática ${ }^{19}$.

0 Estado possui reduzidas responsabilidades, inexistindo uma política mais sólida no que se refere aos papéis atribuídos às famílias e uma rede adequada de suporte a ser oferecida ao idoso e a sua família ${ }^{4}$. A maior dificuldade em desenvolver habilidades que possibilitem a formação de cuidadores mais saudáveis reside no fato de que os cursos ainda são muito voltados para os conceitos e teorias vinculados a uma visão biologicista. Apesar das discussões acerca da importância de se alterar o paradigma no ensino na área da saúde, muitas estratégias precisam ser desenvolvidas na tentativa de se aprimorar as metodologias e também começar a incluir a saúde do cuidador nesse contexto ${ }^{23}$.

Em uma pesquisa em $2003^{24}$ com cuidadores informais de idosos atendidos por um Programa de Saúde da Família (PSF) em Goiás, os autores destacam a necessidade de se conhecer o perfil dos cuidadores informais para a efetiva implantação de programas que envolvam os idosos e suas famílias. Embora o PSF seja uma boa estratégia para o acompanhamento da família, funcionando como porta de entrada do sistema de saúde, ele depende de capacitação adequada dos profissionais incluídos nesse programa.

Pesquisadores em $2002^{16}$ desenvolveram um programa de apoio aos cuidadores no Centro-Escola da Faculdade de Medicina de Botucatu, São Paulo, com o objetivo de promover ações para a preservação da qualidade de vida dos cuidadores familiares através de reuniões semanais com profissionais 
diversos. Essa estratégia se mostrou muito positiva, pois a partir do momento em que o cuidador exteriorizava e compartilhava suas experiências, dúvidas, anseios, ele sentia apoio, reforçando a idéia da necessidade de ampliação de programas desse cunho.

Em um trabalho realizado em $2000^{18} \mathrm{com}$ um grupo de apoio aos cuidadores do NAI/UnATI/UERJ, no Rio de Janeiro, foram destacados importantes objetivos que devem ser introduzidos nos grupos: fazer com que o familiar aceite a irreversibilidade da doença; favorecer a expressão dos sentimentos; dar apoio emocional e prático; permitir a ajuda mútua e facilitar 0 envolvimento do cuidador com o idoso dependente.

Apesar de a temática família estar sendo bastante discutida, pouco se tem estudado sobre a relação do cuidador com o profissional de saúde, sendo mais encontrados estudos que abordam como a família se percebe na sua relação de cuidar $^{25}$. Trabalhar em família não se constitui tarefa fácil e exige a análise acurada do contexto socioeconômico e cultural, no qual se insere a família, analisando suas representações perante a sociedade, conhecendo a sua realidade de forma a desvendar o entendimento da família para que o conhecimento se funda à prática, de forma a superar os limites e possibilidades para a concretização das propostas adequadas ${ }^{26}$.

Não basta apenas preparar um cuidador técnico, torna-se fundamental estruturar as intervenções junto a esse cuidador, que possam ser consideradas também legítimas, de acordo com os anseios morais existentes e resultantes do sentimento de desamparo que afeta concretamente quem cuida desses idosos, muitas vezes sem o suporte necessário ${ }^{27}$.

Diversos trabalhos ${ }^{24}$ destacam a importância das discussões e estudos acerca do significado do cuidado, para que se compreenda o cuidar como um processo que "possui uma dimensão essencial e complexa tanto na experiência de

\section{Referências}

1. Sant'Anna RM, Câmara P,Braga MGC. Mobilidade na terceira idade: como planejar o futuro? Textos sobre Envelhecimento $20032^{\circ} \mathrm{sem} ; 6$ (1): 9-30.

2. Camarano AA, Kanso S, Mello JL. Como vive o idoso brasileiro? In: Camarano AA, organizadora. Os novos idosos brasileiros: muito além dos 60? Rio de Janeiro (RJ): IPEA; 2004. p.25-73.

3. Camarano AA, El Ghaouri SK. Idosos brasileiros: que dependência é essa? In: Camarano AA, organizadora. Muito além dos 60: os novos idosos brasileiros. Rio de Janeiro (RJ): IPEA; 1999. p. 281-304.

4. Karsch UM. Idosos e cuidadores. Cad Saude Publica 2003 mai/ jun; 19 (3): 861-66.

5. Giacomin KC, Uchôa E, Firmo JOA, Lima-Costa MF. Projeto Bambuí: um estudo de base populacional da prevalência e dos fatores associados à necessidade de cuidador entre idosos. Cad Saude Publica 2005 jan/fev; 21 (1): 80-91.

6. Gordilho A, Nascimento JS, Silvestre J, Ramos LR, Freire MPA, Espíndola $\mathrm{N}$, et al. Desafios a serem enfrentados no terceiro milênio pelo setor saúde na atenção integral ao idoso. Rio de Janeiro (RJ): UnATI/UERJ; 2000.

7. Saad PM. Transferências de apoio entre gerações no Brasil: um estudo para São Paulo e Fortaleza. In: Camarano AA, organizadora. Muito além dos 60: os novos idosos brasileiros. Rio de Janeiro (RJ): IPEA; 1999. quem cuida quanto de quem recebe o cuidado ou até mesmo de quem ensina a cuidar e de quem está aprendendo a cuidar".

\section{CONCLUSÃO}

Este estudo abordou a temática "cuidador de idosos dependentes" no contexto da saúde do país. 0 perfil do cuidador constitui-se em uma rede autônoma e geralmente desintegrada dos serviços de saúde, carente de orientações e suporte dos profissionais de saúde.

Ainda permanece a necessidade de programas funcionantes direcionados ao cuidador apesar de toda a legislação vigente, como a Política Nacional de Saúde do Idoso (1999) e o Estatuto do Idoso.

É de grande importância e necessidade a ampliação de ações que tenham o cuidador como sujeito principal, para que essa atividade seja reconhecida e investida em práticas adequadas, trazendo benefícios para quem cuida e quem é cuidado.

Cabe ao profissional de saúde que assiste o idoso dependente ter uma visão mais ampla do processo de envelhecer e da importância da rede de suporte familiar que o idoso possui, com atenção aos idosos sem rede também.

Vale destacar a necessidade de mudanças na forma de atender a essa parcela da população, no tocante ao objetivo das consultas e das ações de cuidado e orientação. Profissionais especializados na área gerontogeriátrica são fundamentais para essa população que aumenta e altera o perfil de adoecimento do país a cada dia.

Estudos e pesquisas são fundamentais para se conhecerem as verdadeiras demandas com o objetivo de formular políticas sociais e econômicas que realmente tenham o idoso como sujeito das ações de saúde.

8. Caldas CP. Cuidado familiar: a importância da família na atenção à saúde do idoso. In: Saldanha AL, Caldas CP, organizadores. Saúde do idoso: a arte de cuidar. $2^{a}$ ed. Rio de Janeiro (RJ): Interciência; 2004. p. 41-7.

9. Giacomin KC, Uchoa E, Lima-Costa MF. Projeto Bambuí: a experiência do cuidado domiciliário por esposas de idosos dependentes. Cad Saude Publica 2005 set/out; 21 (5): 1509-18.

10. Goldani AM. Contratos intergeracionais e reconstrução do estado de bem-estar. Por que se deve repensar essa relação para o Brasil? In: Camarano AA, organizadora. Os novos idosos brasileiros: muito além dos 60? Rio de Janeiro (RJ): IPEA; 2004. p.211-50.

11. Camarano AA, Pasinato MT. 0 envelhecimento populacional na agenda das políticas públicas. In: Camarano AA, organizadora. Os novos idosos brasileiros: muito além dos 60 ? Rio de Janeiro (RJ): IPEA; 2004. p. 253-92.

12. Lavinsky AE, Vieira TT. Processo de cuidar de idosos com acidente vascular encefálico: sentimentos dos familiares envolvidos. Acta Sci Health Sci 2004; 26 (1): 41-5.

13. Cecagno S, Souza MD, Jardim VMR. Compreendendo o contexto familiar no processo saúde-doença. Acta Sci Health Sci 2004; 26 (1): 107-12.

14. Cattani RB, Girardon-Perlini NMO. Cuidar do idoso doente no domicílio na voz de cuidadores familiares. Rev Eletronônica Enferm 2004 [citado 10 dez 2005]; 6 (2). Disponível em: www.fen.ufg.br. 
15. Resta DG, Budó MLD. A cultura e as formas de cuidar em famillia na visão de pacientes e cuidadores domiciliares. Acta Sci Health Sci 2004; 26 (1): 53-60.

16. Cerqueira ATR, Oliveira NIL. Programa de apoio a cuidadores: uma ação terapêutica e preventiva na atenção à saúde dos idosos. Psic-USP 2002; 13 (1).

17. Garrido R, Menezes PR. Impacto em cuidadores de idosos com demência atendidos em um serviço psicogeriátrico. Rev Saude Publica 2004; 38 (6): 835-41.

18. Silveira TM. 0 sistema familiar e os cuidados com pacientes idosos portadores de distúrbios cognitivos. Textos sobre Envelhecimento 2000; $2^{\circ} \operatorname{sem}(3 / 4): 13-28$.

19. Luzardo AR, Waldman BF. Atenção ao familiar cuidador do idoso com doença de Alzheimer. Acta Sci Health Sci 2004; 26 (1): 135-45.

20. Bocchi SCM. Vivenciando a sobrecarga ao vir-a-ser um cuidador familiar de pessoa com acidente vascular cerebral(AVC): uma análise do conhecimento. Rev Latino-am Enfermagem 2004 jan/fev; 12 (1): 115-21.

21. Caldas CP. Envelhecimento com dependência: responsabilidades e demandas da família. Cad Saude Publica 2003 mai/jun; 19 (3): 773-81.

22. Waidman MAP, Elsen I. Família e necessidades... revendo estudos. Acta Sci Health Sci 2004; 26 (1): 147-257.

23. Nakatani AYK, et al. Perfil dos cuidadores informais de idosos com déficit de autocuidado atendidos pelo programa de saúde da familia. Rev Eletrônica Enferm 2003 [citado 15 dez 2005]; 5 (1). Disponível em: www.fen.ufg.br.
24. Damas KCA, Munari DB, Siqueira KM. Cuidando do cuidador: reflexões sobre o aprendizado dessa habilidade. Rev Eletronônica Enferm 2004 [citado 10 dez 2005]; 6(2). Disponível em: www.fen.ufg.br.

25. Lacerda MR, OliniskiSR. O familiar cuidador e a enfermeira: desenvolvendo interações no contexto domiciliar. Acta Sci Health Sci 2004; 26 (1): 239-48.

26. Weirich CF, Tavares JB, Silva KS. 0 cuidado de enfermagem à família: um estudo bibliográfico. Rev Eletronônica Enferm 2004 [citado 10 dez 2005]; 6 (2). Disponível em: www.fen.ufg.br.

27. Floriani CA, Fermin RS. Atendimento domiciliar ao idoso: problema ou solução? Cad Saude Publica 2004 jul/ago; 20 (4): 986-94.

\section{Sobre as Autoras}

\section{Marcia Duarte Moreira}

Enfermeira, Gerontóloga (UERJ), Especialista em Clínica e Cirurgia (UNI RIO).

\section{Célia Pereira Caldas}

Professora da Faculdade de Enfermagem UERJ, Mestre em Saúde Coletiva (IMS-UERJ), Doutora em Enfermagem (EEAN-UFRJ) 